Université de Zagazig

Faculté de Pédagogie

Département de Curricula, de Méthodologie et de Technologie d'enseignement

\title{
Unité proposée basée sur l'apprentissage inversé en vue de développer des compétences de la compréhension lecturale en française, deuxième langue étrangère auprès des étudiants du cycle secondaire
}

\author{
Thèse présentée par \\ Hala Mohammed Mahdy Hagag \\ Sous la surveillance de
}

Prof. Dr. Abdel Fattah Saad Abdel Rahman

Professeur de curricula et de méthodologies de la langue

Française, faculté de pédagogie,

Université de Tanta
Dr. Heba Farouk Nawar

Maître de conférences de curricula et de méthodologies de la langue française, Faculté de pédagogie,

Université de Zagazig 


\section{الجمعية المصرية للقر اعة والمعرفة عضو الجمعية الدولية للمعرفة}

\section{Première partie : cadre générale de la recherche}

\section{Introduction}

La langue est un outil essentiel de communication, elle aide l'homme à communiquer, à exprimer ses besoins, ses pensées, ses sentiments, ses ambitions et à entrer en contact avec les autres. Il est nécessaire pour l'individu d'apprendre plus qu'une langue étrangère qui pour communiquer avec les autres individus et reconnaître les différentes civilisations.

L'enseignement d'une langue étrangère vise non seulement à développer la capacité de l'apprenant à communiquer en cette langue, mais aussi à développer ses facultés intellectuelles, affectives et sensorielles afin de le rendre apte à imaginer, à découvrir, à inventer, à s'exprimer et à construire son propre savoir (Youssef, A., 2011,1).*

La connaissance d'une langue étrangère suppose l'acquisition de quatre compétences langagières (écouter, parler, lire, écrire).

En effet, l'enseignement/apprentissage d'une langue étrangère doit prendre en considération ces quatre compétences comme unité intégrale sans négliger l'une ou l'autre.

Dans ce domaine, la lecture occupe une place distinguée parmi ces quatre compétences ou elle constitue le centre de deux compétences (écouter, parler) et le début de la dernière phase de la pyramide linguistique (Abdel hamed, R., 2014, 391). 


\section{الجمعية المصرية للقر اءة والمعرفة عضو الجمعية الدولية للمعرفة ILA}

* La documentation est d'après le système suivant: Nom de l'auteur, son prénom, la date, la page.

Malgré l'extension de plus en plus grande que prennent la télévision la radio, et la technologie moderne, la lecture est considérée le plus important moyen de culture, la source la plus riche d'informations scientifiques et culturelles.

La lecture constitue une partie très importante dans l'enseignement d'une langue étrangère parce qu'elle a une grande influence sur la connaissance de la langue sur, l'acquisition du vocabulaire et sur les progrès grammaticaux et orthographe (El seify, M., 2009, 3).

A cet égard, la lecture joue un grand rôle dans le processus de l'enseignement/ apprentissage. Puis qu'elle aide les étudiants à élargir et à développer leurs expériences, à animer leurs habiletés intellectuelles, à satisfaire leurs gouts, à sonder les autres et à s'introduire dans l'actualité internationale (Bahi El din, M., 2016, 5).

De plus, il n'y a pas de lecture sans compréhension, l'étudiant utilise quelques compétences et activités pour interpréter ce qui est écrit et arriver au sens de texte lu et comprendre son message (Tantawy, N., 2018, 3).

Il existe quatre niveaux de compréhension en lecture : 


\section{الجمعية المصرية للقر اعة والمعرفة عضو الجمعية الدولية للمعرفة ILA}

-Compréhension littérale: c'est comprendre les informations ou les idées données de façon explicite par l'auteur dans un texte. Cette compréhension représente la base de tous les autres niveaux de compréhension.

- Compréhension inferentielle ou interprétative: c'est comprendre des informations supplémentaires.

-Compréhension critique: c'est porter un jugement sur le texte lu. -Compréhension créative: c'est le niveau de compréhension qui permet au lecteur de créer de nouvelles idées.

La compréhension en lecture a une place primordiale parmi les autres compétences langagières. Malgré son intérêt, elle reste encore le point majeur de faiblesse chez la majorité des apprenants. Cette remarque a été consultée par plusieurs études antérieures: (Tantawy, N., 2018), (El Bahnassy, L., 2016), (Abdel-aal, M., 2016), (Abdel hamed, R., 2014), (Gaafar, A., 2011), (Attia, H., 2010), (Sabri, N., 2009), (Ezz el arab, M., 2003), (Chérif, A., 2002), (Achri, A., 1987).

Autrement dit, toutes ces études ont souligné que le développement de la compréhension en lecture est une nécessité parce qu'elle aide les étudiants à:

1-fortifier leur inspiration.

2- cultiver leur imagination. 
3- appliquer et communiquer leurs connaissances antérieures et leurs compétences dans de nouveaux contextes.

Ainsi, les études antérieures ont conseillé l'usage des stratégies modernes, efficaces, et appropriées. Parmi les quelles la stratégie de l'apprentissage inversé, qui a un effet positif sur le développement des compétences de la compréhension lecturale comme: (Saad, D., 2019), (Chehata, M., 2019), (Ibrahim, W., 2018), (El Delami, G., 2017), (Manon, A., 2016).

Il est évident que le monde, où nous vivons, est devenu un petit village et se caractérise par le changement technologique et le développement des sciences pédagogiques qui, à leur tour, ont des influences sur l'apprenant.

Pour ces raisons, on doit former une génération d'étudiants capables de

s'adopter aux nouveaux changements dans les différents domaines (Abdel hamed, R., 2014, 3).

A cet égard, nous vivons dans l'époque de technologie; pour cela il est important d'utiliser de nouvelles stratégies, qui convient avec la révolution technologique. Parmi ces stratégies, on peut distinguer l'apprentissage inversé. La chercheuse a choisi cette stratégie qui accorde plus de temps de manipulation avec l'enseignant et lui 


\section{الجمعية المصرية للقر اعة والمعرفة عضو الجمعية الدولية للمعرفة ILA}

permet de guider les étudiants au moment de l'assimilation de l'information et de la création de nouvelles idées.

L'apprentissage inversé c'est l'une des nouvelles stratégies dans le domaine de la didactique des langues étrangères, c'est un modèle pédagogique qui met à profit l'apprentissage à distance (hors classe) pour renforcer l'engagement, l'implication et l'apprentissage des étudiants en classe. Les activités qui réclament des habiletés cognitives de haut niveau, c'est-à-dire les objectifs d'apprentissage plus complexes, sont réalisées en classe, en mettant l'accent sur le travail en groupe et l'apprentissage collaboratif, alors que les activités sollicitant des habiletés de bas niveau cognitif sont réalisées à distance, en dehors de la classe (Chehata, M., 2019, 19).

Selon (Lebrun, M., 2015, 73), l'apprentissage inversé consiste à déplacer la partie magistrale d'un cours à la maison, et à utiliser le temps de classe pour réaliser les devoirs traditionnellement faits à la maison.

Dufour, H. (2014, 45) a souligné que l'apprentissage inversé permettrait d'individualiser et de différencier l'enseignement du fait que l'apprenant travaille individuellement et à son rythme hors classe (à la maison). Ce même auteur énonce également que cette forme d'enseignement favoriserait le tutorat par les pairs. Selon elle, lors des travaux de groupe en salle de classe, « les élèves qui ont en difficulté bénéficient du soutien et des explications de leurs pairs, 


\section{الجمعية المصرية للقر اعة والمعرفة عضو الجمعية الدولية للمعرفة}

tandis que les élèves qui ont à l'aise, en expliquant à leurs pairs, approfondissent leur compréhension et renforcent leur apprentissage $\gg$.

L'apprentissage inversé, est une stratégie pédagogique où la partie transmissive de l'enseignement (exposé, consignes, protocole, etc.) se fait «à distance » en préalable à une séance en présence, notamment à l'aide des technologies (vidéo en ligne du cours, lecture de documents papier, préparation d'exercice, etc.) et où l'apprentissage fondé sur les activités et les interactions se fait « en présence» (Lebrun, M et Lecoq, J., 2015, 16).

Il existe plusieurs types de classes inversées. Lebrun, M. (2017, 7) en distingue trois:

- Le premier, c'est le plus « classique », il se base sur la préparation de la leçon à la maison et les devoirs en classe. Le « savoir » est externalisé à l'aide du numérique, alors que des activités pour accompagner l'apprentissage sont prévues en classe.

- Le deuxième, l'apprenant et l'enseignant échangent rôle. Les apprenants

cherchent eux-mêmes les savoirs et, de retour en classe, ils présentent les

résultats de leur recherche ou préparent une activité avec leurs camarades. 


\section{الجمعية المصرية للقراعة والمعرفة عضو الجمعية الدولية للمعرفة ILA}

- Le troisième combine les deux types précédents en alternant des différents types d'activités.

De tout ce qui est déjà mentionné, on peut déterminer les objectifs l'apprentissage inversé comme suit: (Hamdan, $\mathbf{N}$ et

\section{McKnight, P., 2013)}

- Développer l'autonomie des élèves.

- Responsabiliser les élèves dans leur apprentissage.

- Développer le travail collaboratif.

- Développer les compétences individuelles des élèves.

- Utiliser tous les medias possibles pour faciliter l'apprentissage.

- Différencier la pédagogie: l'objectif est que l'élève progresse selon son propre rythme.

- Améliorer les résultats scolaires d'élève.

- Personnaliser le travail de l'élève.

- Impliquer et motiver l'élève pour sa formation.

- Utiliser les outils modernes.

\section{- Problématique de la recherche:}

Le problème de cette recherche réside dans la faiblesse du niveau des étudiants de la deuxième année secondaire dans les compétences de la compréhension lecturale, ce qui exige l'utilisation 


\section{الجمعية المصرية للقر اعة والمعرفة عضو الجمعية الدولية للمعرفة ILA}

de nouvelles stratégies plus actives et efficaces pour développer ces compétences.

Cette recherche a essayé de répondre à la question principale suivante: "Quelle est l'efficacité de l'apprentissage inversé pour développer quelques compétences de la compréhension en lecture française, deuxième langue étrangère auprès des étudiants du cycle secondaire?"

Pour répondre à cette question, on doit répondre aux questions secondaires suivantes:

1-Quelles sont les compétences de la compréhension en lecture nécessaires aux étudiants de la deuxième année secondaire (échantillon de la recherche)?

2-Jusqu'à quel niveau les étudiants de la deuxième année secondaire maitrisent-ils ces compétences?

3-Quelle est la forme de l'une unité reformulée à la lumière des principes philosophiques de la stratégie de l'apprentissage inversé?

4- Quelle est l'efficacité de l'unité, déjà citée, dans le développement de quelques compétences de la compréhension en lecture en français auprès de l'échantillon visé?

\section{- Objectifs de la recherche:}

Cette recherche vise à: 


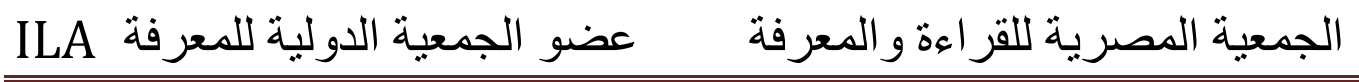

1-Développer les compétences de la compréhension lecturale en français, langue étrangère auprès des étudiants du cycle secondaire. 2-Utiliser la stratégie de l'apprentissage inversé pour développer quelques compétences de la compréhension lecturale auprès des étudiants du cycle secondaire.

3-Mesurer l'efficacité de la stratégie de l'apprentissage inversé pour développer quelques compétences de la compréhension en lecture en français auprès des étudiants de l'échantillon de cette recherche.

\section{- Importance de la recherche:}

Cette étude pourrait être profitable pour :

1-Les apprenants: développer quelques compétences de la compréhension lecturale auprès des étudiants du cycle secondaire.

2- Les enseignants : Cette recherche pourrait fournir aux enseignants du français des informations sur de nouvelles stratégies dans le domaine de la didactique du français où la stratégie de l'apprentissage inversé encourage les enseignants à améliorer le processus d'enseignement.

3-Les chercheurs : cette recherche pourrait ouvrir de nouveaux horizons pour les chercheurs à effectuer des études et des recherches pour développer quelques compétences de la compréhension lecturale dans d'autres cycles d'apprentissage en utilisant de nouvelles méthodes et stratégies qui ont mis l'accent sur les apprenants positifs. 


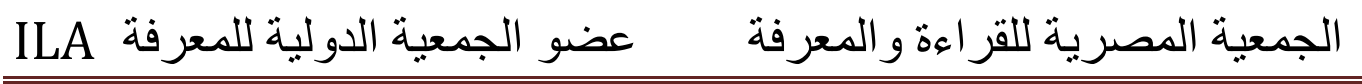

4- Les responsables du programme : cette étude peut attirer

l'attention des responsables, qui préparent des programmes pour les étudiants du cycle secondaire, à s'intéresser au développement des compétences de la compréhension en lecture auprès des étudiants du cycle secondaire.

\section{- Délimitations de la recherche:}

Cette recherche se limite à:

1-Un échantillon des étudiants de la deuxième année secondaire. Ces étudiants ont déjà étudié le français en 1ère année secondaire et ont un bon bagage langagier qui leur permet de développer leurs compétences de la compréhension lecturale.

2-Quelques compétences de la compréhension lecturale qui conviennent au niveau linguistique et intellectuel des étudiants de la deuxième année secondaire.

3- La stratégie de l'apprentissage inversé.

4- Une unité reformulée à la lumière des principes

philosophiques de la stratégie de l'apprentissage inversé du manuel

\section{"Club@dos plus 2".}

\section{- Hypothèses de la recherche:}

1- Il y a une différence statistiquement significative au niveau de (0.01) entre les moyennes des notes des étudiants des deux groupes (expérimental, témoin) au post-test des 


\section{الجمعية المصرية للقر اعة و المعرفة عضو الجمعية الدولية للمعرفة ILA}

étudiants du groupe expérimental en faveur du groupe expérimental.

2- Il y a une différence statistiquement significative au niveau de (0.01) entre les moyennes des notes des étudiants du groupe expérimental au pré/post-test de la compréhension lecturale en faveur du post-test.

3- L'apprentissage inversé a un effet pour développer les compétences de la compréhension lecturale auprès des étudiants du groupe expérimental.

\section{- Méthodologie de la recherche:}

La chercheuse a adopté deux méthodes:

\section{1-La méthode descriptive:}

Pour réviser les études antérieures concernant le problème et les variables de la recherche.

\section{2-La méthode expérimentale:}

Pour mesurer l'efficacité de l'apprentissage inversé dans le développement des compétences de la compréhension lecturale auprès des étudiants de la deuxième année Secondaire.

\section{3-Le plan expérimental:}

La chercheuse a utilisé le plan expérimental des deux groupes: témoin et expérimental en appliquant un pré/post test.

\section{- Procédures de la recherche:}




\section{الجمعية المصرية للقر اعة والمعرفة عضو الجمعية الدولية للمعرفة}

Pour répondre aux questions de cette recherche et vérifier ses hypothèses, la chercheuse a suivi les procédures suivantes :

1- Passer en revue les recherches et les études antérieures concernant les deux axes principaux de l'étude théorique: la compréhension en lecture et la stratégie de l'apprentissage inversé; afin de bien comprendre leurs concepts, leurs composantes, leur nature...etc.

2- Elaborer un questionnaire de compétences de la compréhension lecturale aux étudiants de la deuxième année secondaire.

3- Présenter la liste de compétences dans sa forme initiale au jury des spécialistes du FLE,

4- Modifier cette liste à la lumière des points de vue du jury.

5- Elaborer un pré-post test de la compréhension lecturale afin de mesurer

quelques compétences de la compréhension lecturale en français auprès les

membres de l'échantillon, ce qui permet de vérifier l'efficacité de la stratégie de l'apprentissage inversé.

6- Présenter ce test dans sa forme initiale aux membres du jury puis le modifier d'après leurs avis.

7-Choisir une unité du manuel de la $2^{\text {éme }}$ année secondaire et la reformuler selon la philosophie de l'apprentissage inversé. 


\section{الجمعية المصرية للقر اعة والمعرفة عضو الجمعية الدولية للمعرفة ILA}

8- Présenter cette unité au jury et y faire les modifications nécessaires.

9- Choisir l'échantillon de la recherche parmi les étudiants de la deuxième année, secondaire et le répartir en deux groupes: expérimental (30 étudiants) et témoin (30 étudiants).

10-Appliquer le pré - test de la compréhension en lecturale aux membres des deux groupes: expérimental et témoin.

11- Enseigner l'unité reformulée à la lumière des principes philosophiques de la stratégie de l'apprentissage inversé aux étudiants du groupe expérimental et procéder la méthode en cours suivie au groupe témoin.

12-Appliquer le post - test de la compréhension lecturale aux étudiants des deux groupes: expérimental et témoin.

13-Analyser statiquement les notes des étudiants, interpréter les résultats et proposer quelques recommandations et suggestions.

\section{- Terminologies de la recherche}

1-L'apprentissage inversé: c'est un des modèles d'apprentissage intégrés, dans lequel l'environnement d'apprentissage est reflété d'une façon plus utile, où les apprenants visionnent des vidéos avant de venir en cours pour modifier le temps du cours, et l'utiliser pour répondre aux questions des étudiants, résoudre des problèmes et expliquer des concepts difficiles, en les intégrant dans un 


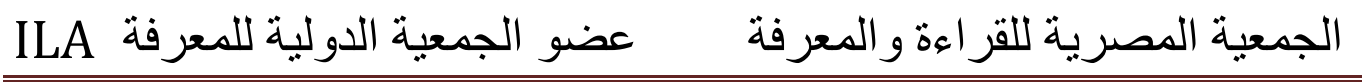

apprentissage efficace et en reliant l'apprenant à son environnement et à la vie quotidienne (Fluton, K., 2014, 67).

2-La compréhension en lecture: Giasson, J. (2000, 2-15) a défini la compréhension lecturale comme "acquérir le vocabulaire et les connaissances nécessaires pour comprendre les textes, dégager le thème d'un paragraphe ou d'un texte court et comprendre de mots nouveaux, de phrases, de textes informatifs, documentaires et littéraires".

\section{Deuxième partie : étude expérimentale}

Dans cette partie, on va présenter les procédures suivies pour préparer les outils et les étapes de l'expérimentation de la recherche afin de vérifier l'efficacité de l'apprentissage inversé pour développer les compétences de la compréhension lecturale en français auprès des étudiants de la deuxième année au cycle secondaire. Ces procédures sont:

\section{I-Elaboration des outils et des matériels de la recherche.} A. Un questionnaire des compétences de la compréhension lecturale en FLE.

La forme initiale du questionnaire contient (4) compétences principales et (24) compétences secondaires, présentées cidessous:

- Compétences de la compréhension littérale. 


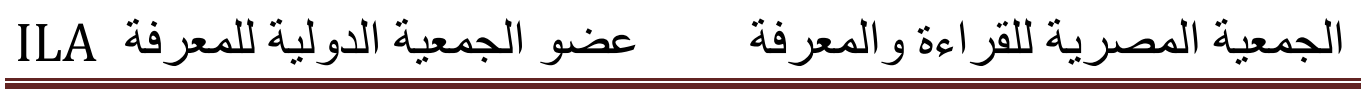

\section{- Compétences de la compréhension inférentielle ou} interprétative.

- Compétences de la compréhension critique.

-Compétences de la compréhension créative.

B. Une liste des compétences de la compréhension lecturale en FLE.

On a abouti une liste des compétences de la compréhension lecturale en FLE, ayant tiré en considération les remarques du jury sur la forme initiale du questionnaire des compétences de la compréhension lecturale. Donc, la liste des compétences est composée de (17) compétences de la compréhension lecturale.

C. Un test des compétences de la compréhension lecturale en FLE.

Ce test se compose de 7 questions qui testent le niveau des étudiants de la deuxième année en compétences de la compréhension lecturale, ayant en totalité de notes (50), les questions sont variées.

D. Une unité reformulée en utilisant l'apprentissage inversé.

On a choisi la quatrième unité de la série Club@ dos plus 2, consacrée aux étudiants de la deuxième année secondaire au deuxième semestre pour les raisons suivantes : 
-Cette unité est riche, il comporte un vocabulaire, des expressions, et des idées. Cela nous permet de développer les compétences de la compréhension lecturale auprès de l'échantillon.

Cette unité reformulée "le club des décorateurs" comporte trois leçons présentées sous forme des feuilles du travail:

\begin{tabular}{|l|l|}
\hline Leçon(1) & Parler des activités quotidiennes. \\
\hline Leçon(2) & Comparer et dire ce qu'on préfère. \\
\hline Leçon(3) & Demander de faire (ou non)quelque chose. \\
\hline
\end{tabular}

\section{II-Précision des étapes de l'expérimentation de la recherche.}

\section{A. Choix de l'échantillon de la recherche.}

La chercheuse a choisi un groupe des étudiantes par hasard (30) de la deuxième année du cycle secondaire qui étudient le français comme deuxième langue étrangère; les étudiantes ont des connaissances antérieures en français parce qu'elles l'ont déjà étudié pendant leur première année secondaire.

\section{B. Application du pré-test de la compréhension en lecture.}

Avant l'enseignement de l'unité reformulée, le test a été appliqué le 16/2/2020 et a duré $\mathbf{5 0}$ minutes sur les membres de l'échantillon de la recherche. 


\section{Enseignement de l'unité reformulée d'après les étapes de} l'apprentissage inversé.

-Après avoir appliqué le pré-test de la compréhension en lecture, la chercheuse a commencé l'application de l'unité sur le groupe expérimental.

-L'expérimentation de cette unité reformulée a commencé le 17/2/2020 et a terminé le 8/2/2020. L'application de cette unité s'est déroulée au deuxième semestre de l'année scolaire 2019/ 2020.

\section{Application du post-test de la compréhension en lecture.}

Après avoir terminé l'application de l'unité reformulée, qui a duré 50 minutes, nous avons présenté les post-tests aux membres de deux groupes (témoin et expérimental) en vue de mesurer l'efficacité de l'unité reformulée d'après les principes philosophiques de l'apprentissage inversé. Nous avons corrigé les réponses des étudiants selon les critères de notation déjà déterminés. Nous avons calculé les moyennes et les écarts types pour les utiliser à l'analyse statistique.

\section{Troisième partie : résultats de la recherche}

Pour analyser les résultats du pré-post test, le chercheur a utilisé le test de (T) selon le programme SPSS. 


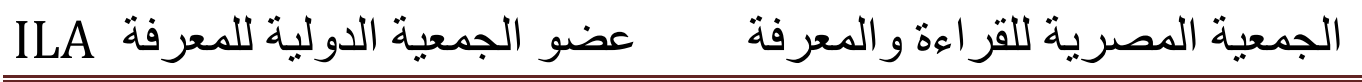

Tableau no. (2): signification de l'application du pré/posttest de la compréhension lecturale auprès des membres des deux groupes.

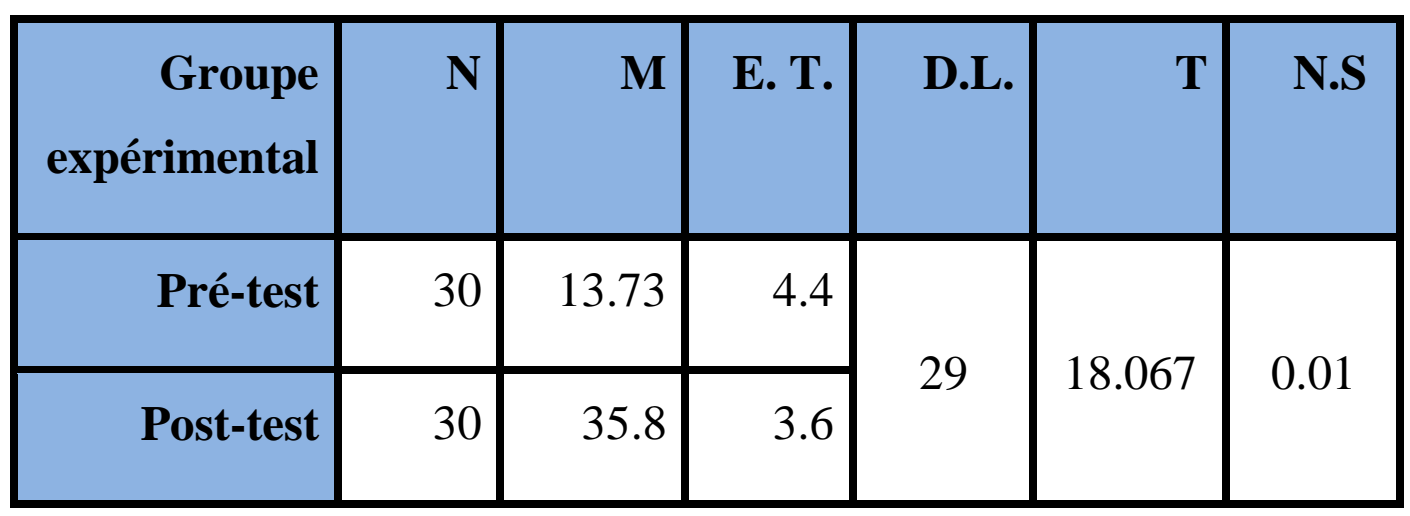

Selon les données du tableau déjà exposé, on peut observer que la valeur de la signification est égale à (0.00). Cette valeur est inférieure au niveau de signification (0.01). Cela signifie qu'elle est significative. Ce qui confirme qu'il y a une différence statistiquement significative au niveau de (0.01) entre des étudiants du groupe expérimental au pré/post-test de la compréhension lecturale français en faveur du post-test qui est le plus supérieur aux moyennes des notes où les moyennes des notes des membres du groupe expérimental à la post-application étaient(35.8)et les moyennes de leurs notes au pré-test est (13.73). Cela signifie que le niveau des étudiants du groupe expérimental au post-test des compétences de la compréhension

$$
\text { r. }
$$




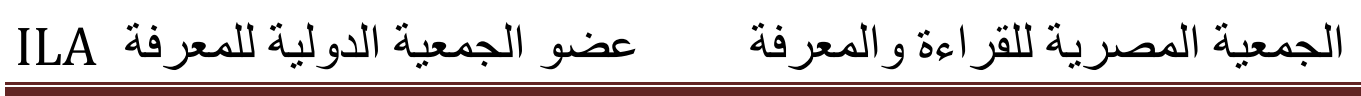

lecturale est supérieur à celui du pré-test. Donc la première hypothèse est confirmée.

Tableau no. (2): signification des notes de l'application du post-test de la compréhension lecturale.

\begin{tabular}{|c|c|c|c|c|c|c|}
\hline Groupe & $\mathbf{N}$ & $\mathbf{M}$ & E. T. & D.L. & $\mathbf{T}$ & N.S. \\
\hline Témoin & 30 & 13.8 & 3.53 & & & \\
\cline { 1 - 5 } Expérimental & 30 & 35.8 & 3.61 & 58 & 23.83 & 0.01 \\
\hline
\end{tabular}

Les résultats du tableau précédent montrent que la valeur de signification est égale à (0.00). Cette valeur est inférieure au niveau de signification (0.01). Ce qui confirme qu'il y a une différence statistiquement significative au niveau de (0.01) entre étudiants des deux groupes (expérimental, témoin) au post-test de la compréhension lecturale en français en faveur des étudiants du groupe expérimental où les moyennes des notes des membres du groupe expérimental au post application est (35.8) et les moyennes des notes des membres du groupe témoin est (13.8). Cela signifie que le niveau des étudiants du groupe expérimental au post-test des compétences de la compréhension lecturale est 


\section{الجمعية المصرية للقر اعة والمعرفة عضو الجمعية الدولية للمعرفة ILA}

supérieur à celui niveau des étudiants du groupe témoin. Donc, la deuxième hypothèse a été vérifiée.

\section{- Calculer la taille d'effet de l'emploi de}

\section{l'apprentissage inversé}

La chercheuse a calculé la taille d'effet de l'emploi de l'apprentissage inversé sur le développement des compétences de la compréhension lecturale en FLE auprès l'échantillon de la recherche selon l'équation suivante:

La valeur d'Eta $\left(\eta^{2}\right)=\frac{T^{2}}{T^{2+D . L}}$

Tableau no. (3): taille de l'effet de l'emploi de la stratégie de l'apprentissage inversé sur le développement des compétences de la compréhension lecturale en FLE auprès l'échantillon de la recherche.

\begin{tabular}{|c|c|c|r|r|r|r|}
\hline $\begin{array}{c}\text { Variable } \\
\text { indépendante }\end{array}$ & $\begin{array}{c}\text { Variable } \\
\text { dépendante }\end{array}$ & $\mathbf{T}$ & $\mathbf{T}^{2}$ & & $\left(\boldsymbol{\eta}^{2}\right)$ & T. E. \\
\hline $\begin{array}{c}\text { Apprentissage } \\
\text { inversé }\end{array}$ & $\begin{array}{c}\text { Compréhension } \\
\text { Lecturale }\end{array}$ & 18.067 & 326.4 & 29 & 918.0 & grande \\
\hline
\end{tabular}

Selon les résultats du tableau déjà exposé, on peut observer que la taille de l'effet de l'emploi de la stratégie de 


\section{الجمعية المصرية للقراءة والمعرفة عضو الجمعية الدولية للمعرفة ILA}

l'apprentissage inversé sur le développement des compétences de la compréhension lecturale en FLE auprès l'échantillon de la recherche est (0.918) plus de (0.20), ce qui montre que la taille de l'effet est grande. Cela signifie que l'effet de la variable indépendante (l'apprentissage inversé) sur la variable dépendante (compétences de la compréhension lecturale) est très grand. Ces valeurs indiquent qu'il y a une efficacité de l'apprentissage inversé sur le développement des compétences de la compréhension lecturale auprès les étudiants de la deuxième année secondaire. Par conséquent, la troisième hypothèse est aussi vérifiée.

$>$ Recommandations de la recherche :

À la lueur des résultats obtenus de cette recherche, on peut recommander de /d':

- Mettre les étudiants au centre du processus d'apprentissage et non seulement des récepteurs d'information.

- Encourager les apprenants à 'être plus autonomes en développant leurs compétences linguistiques.

- Motiver les étudiants à communiquer en langue étrangère à travers des situations de la vie quotidienne et mobiliser leurs capacités à s'exprimer entre eux avec des phrases simples et correctes. 


\section{الجمعية المصرية للقر اعة و المعرفة عضو الجمعية الدولية للمعرفة ILA}

- Utiliser des méthodes modernes appliquées dans le domaine de l'apprentissage des langues étrangères qui reposent sur l'emploi du support audio-visuel (audio - vidéo - dessin image - cartes etc...). La variété des techniques aide les apprenants à écouter et à communiquer efficacement.

$>$ Suggestions de la recherche :

En reposant sur les résultats obtenus de cette

recherche, on peut suggérer les thèmes suivants à chercher:

1) Vérifier l'efficacité de la stratégie de l'apprentissage inversé pour développer les compétences de la compréhension en lecture pour tous les cycles éducatifs.

2) Utiliser l'apprentissage inversé pour développer d'autres compétences de la langue auprès des apprenants de tous les cycles éducatifs.

3) Faire des comparaisons entre la stratégie d'apprentissage inversé et d'autres stratégies d'enseignement et mesurer leur efficacité à développer les compétences de la compréhension lecturale.

\section{- Bibliographie}

\section{A) Références en langue francaise:}

1. Abdel-aal, Mohammed (2016)."Éfficacité de la stratégie de l'enseignement réciproque pour le 
développement des compétences des textes lus et l'attitude envers la langue française chez les étudiants du cycle secondaire d'Al Azhar". Thèse de magistère, faculté de pédagogie, université de Mansourah.

2. Abdel hamed, Rasha (2014)."Éefficacité de l'emploi de la stratégie des cartes sémantiques sur le développement des compétences de la compréhension lecturale chez les étudiants de la faculté de pédagogiesection de français". Thèse de magistère, faculté de pédagogie, université de Mansourah.

3. Abdel hamed, Achri (1987)."La lecture suivie en français au cycle secondaire en Egypte". Thèse de doctorat, faculté de pédagogie, université d'AinChams.

4. Attia, Heba (2010)."Éfficacité de l'apprentissage coopératif sur le développement de quelques compétences de la compréhension lecturale en français chez les étudiants du cycle secondaire de langues". Thèse de magistère, faculté de pédagogie, université de Mansourah.

5. Bahie El Din, Mona (2008)."Éfficacité d'un programme propose en vue de développer les compétences de la lecture silencieuse et de la pensée 
critique chez les futurs enseignants de français, Thèse de magistère, Faculté de jeunes filles, Université d'Ain chams.

6. Chérif, Abdel nasser (2002)."Modèle proposé pour le développement des compétences de la lecture créative en français langue étrangère chez l'étudiant-maître." Thèse de Doctorat, faculté de jeunes filles, Université d'Ain-Chams.

7. Chehata, Marwa (2019). " Efficacité de la stratégie des classes inversées dans l'enseignement de la langue française pour développer quelques compétences du $21^{\mathrm{e}}$ siècle chez les étudiants du cycle secondaire." Thèse de Doctorat, faculté de pédagogie, Université d'El Sadat.

8. Dufour, Héloïse (2014).La classe inversée. Revue technologie Eduscol, (193), 44-47.

9. El seify, Maha (2005)."Emploi de l'enseignement réciproque en vue de développer les compétences de la compréhension en lecture nécessaires aux étudiants de la faculté de pédagogie". Thèse de magistère, faculté de pédagogie, université de Ménoufya.

10. Gafaar, Amal (2011)."Utilisation des cercles de lecture littéraire pour développer quelques compétences de la compréhension lecturale en langue français chez 
les élèves du cycle primaire". Thèse de Magistère,

Faculté de Pédagogie, Université d'Ain-Chams.

11. Ibrahim,Walaa (2018)."Éfficacité de l'utilisation de la stratégie de l'enseignement inversé sur le développement des compétences langagières en français chez les étudiants des écoles de langues-cycle secondaire". Thèse de magistère, Faculté de pédagogie, Université de Mansourah.

12. Lebrun, Marcel. \& Lecoq, Julie. (2015). Classes inversées, Enseigner et apprendre à l'endroit ! Maîtriser, Réseau Canopé.

13. Lebrun, Marcel. (2014). Classes inversées, étendons et —systémisons\| le concept ! : Essai de modélisation et de systémisation du concept de Classes inversées [billet de blog]. Récupéré sur http://lebrunremy.be/WordPress/?p=740.

14. Lebrun, Maecel. (2015). L'hybridation dans l'enseignement supérieur : vers une nouvelle culture de l'évaluation? Evaluer. Journal international de Recherche en Education et Formation, 1(1), p.65-78. Récupéré de http://ejref. org/ index. php? id=91\&file=1 
15. Lebrun, Marcel. \& Lecoq, Julie. (2017). La classe à l'envers pour apprendre à l'endroit. Louvain-la-Neuve : Louvain Learning Lab (LLL).

16. Manon, Aurélie (2016). Quels sont les effets de la classe inversée sur les performances des élèves? Master Métiers de l'enseignement, de l'éducation et de la formation, Ecole supérieure du professorat et de l'éducation, Université Savoie Mont Blanc.

17. Saad, Dalia (2019). " Efficacité de la stratégie d'apprentissage inversé pour développer les compétences de la communication orale auprès des étudiants de la faculté de pédagogie section de français". Thèse de magistère, faculté de pédagogie, Université de Ménoufia.

18. Sabri, Nermin (2009)."Éfficacité d'un programme propose á la lueur de l'approche Histoires sur le développement de quelques compétences de la compréhension lecturale en langue française et de valeurs morales chez les élèves du cycle préparatoire". Thèse de Doctorat, Faculté de Jeunes Filles, Université d'Ain-Chams. 
19. Tantawy, Noura (2018)."Éfficacité de l'apprentissage autorégulé pour développer quelques compétences de la compréhension lecturale en français chez les étudiants du cycle secondaire". Thèse de magistère, faculté de pédagogie, université de Mansourah.

20. Youssef, Ahmed (2011)."Éfficacité d'un programme basé sur les stratégies d'apprentissage sur le développement des compétences de la compréhension en lecture et de l'expression écrite créative chez les étudiants de la section de français, faculté de pédagogie, université d'Al Azhar". Thèse de doctorat, faculté de pédagogie, université d'Al Azhar.

21. El bahnassy, Laila (2016)."Utilisation de l'hypertexte pour développer quelques compétences de la compréhension écrite chez les étudiants du département de français à la faculté de pédagogie de Tanta". Thèse de magistère, Faculté de Pédagogie, Université de Tanta.

\section{B) Références en langue anglaise:}

1- Hamdan, Noora\& McKnight, Patrick\& McKnight, Katherine\& Arfstrom, Kari (2013).A review of flipped learning: George Mason University. Retrieved 


\section{ILA الجمعية المصرية للقر اعة والمعرفة عضو الجمعية الدولية للمعرفة}

10January2018from:http://www.flippedlearning.org/cms/l ib07/VA01923112/Centricity/Domain/41/LitReview-

FlippedLearning.pdf.

\section{C) Références en langue arabe:}

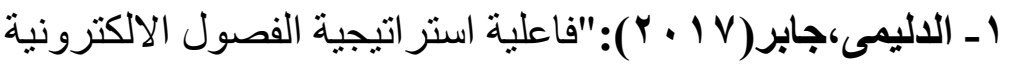

المعكوسة فى تتمية بعض نو اتج التعلم المهارية لدى طلاب المرحلة

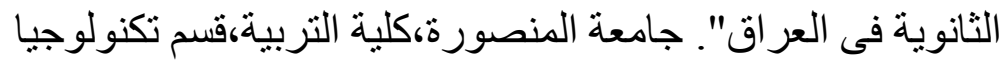

التعليم 